Although Perrotti et al. had expected the number of claims to rise over the study period, the number of claims remained constant at an average of 22 per year. They found, however, that the average indemnity payment rose by almost $10 \%$ each year; after adjusting for inflation, these payments almost tripled over the study period.

The authors acknowledge that insurance companies face many more costs than simply indemnity payments; nevertheless, they propose that the increase in indemnity payments is a major contributory factor to the rise in malpractice insurance premiums.

Original article Perrotti M et al. (2006) Medical malpractice in urology, 1985 to 2004: 469 consecutive cases closed with indemnity payment. J Urol 176: 2154-2157

\section{The efficacy of organ-sparing therapies for penile cancer}

The traditional treatment for squamous-cell carcinoma of the penis is partial or total penectomy, which causes the patient considerable psychological distress. Organ-sparing treatments would be preferable to the patient, but there is a lack of randomized studies that compare the outcomes of the available options. A team of Swiss and French researchers have conducted a retrospective study of men who received various treatments for penile cancer.

Ozsahin et al. analyzed the records of 60 consecutive patients with nonmetastatic, invasive squamous-cell carcinoma of the penis, who were treated between 1962 and 1994. Overall, 27 patients underwent partial or total penectomy, 8 patients received brachytherapy, 21 patients received primary external radiotherapy, and 4 patients refused radiotherapy after excisional biopsy; 22 of 27 penectomy patients and 7 of 8 brachytherapy patients also received postprocedure external radiotherapy.

Local failure was more common in patients who had undergone organ-sparing procedures than in patients who had undergone penectomy (56\% versus $13 \%, P=0.0008$ ); however, no difference in survival was seen between penectomy patients and patients who had received primary radiotherapy. The authors propose that primary radiotherapy is a reasonable treatment option, if patients are closely followed up. It should be noted that, because of the retrospective design of the study, it was not possible to assess penile function, which might be affected even by organ-sparing treatment.

Original article Ozsahin M et al. (2006) Treatment of penile carcinoma: to cut or not to cut? Int J Radiat Oncol Biol Phys 66: 674-679

\section{Lymphatic staining to prevent hydrocele after laparoscopic varicocele ligation}

Laparoscopic ligation is the favored treatment for varicocele in children and adolescents. A common postoperative complication of varicocele surgery is the disruption of testicular lymphatic drainage, which results in hydrocele. Visualization of the lymphatic vessels by dye injection reduces the incidence of hydrocele after open Palomo ligation; Schwentner et al., therefore, evaluated whether lymphatic staining was feasible during laparoscopic ligation.

The authors enrolled 50 consecutive patients (aged 8-25 years) with varicocele, confirmed by scrotal color Doppler ultrasonography. The patients were randomly allocated to laparoscopic varicocele ligation only, or laparoscopic varicocele ligation following injection of $2 \mathrm{ml} 1 \%$ isosulphan blue (under the tunica dartos, near the parietal wall of the tunica vaginalis) $15 \mathrm{~min}$ before surgery. The patients were followed up postoperatively at 3, 6, and 12 months and annually thereafter.

Lymphatic staining was successful in $24 / 25$ patients. No patients allocated to the isosulphan-blue group developed hydrocele, but two patients had a blue-stained scrotum at 3 months that had resolved by the 6 month checkup. Five patients who underwent laparoscopic ligation without lymphatic staining had secondary hydroceles at 3 months, which were still present 6 months after surgery (three underwent hydrocelectomy 1 year after surgery). Varicocele persisted in two patients (one from each treatment group). The mean operative time was similar in both groups.

The authors conclude that preservation of adequate lymphatic drainage is essential to avoid postoperative hydrocele and improve the andrologic outcome of varicocele surgery. They recommend use of lymphatic staining to ensure lymphatic vessels are spared.

\footnotetext{
Original article Schwentner C et al. (2006) Laparoscopic varicocele ligation in children and adolescents using isosulphan blue: a prospective randomized trial. BJU Int 98: 861-865
} 